\title{
Risk factors for developing dementia in type 2 diabetes mellitus patients with mild cognitive impairment
}

This article was published in the following Dove Medical Press journal: Neuropsychiatric Disease and Treatment

\section{Oana Albai' \\ Mirela Frandes ${ }^{2}$ \\ Romulus Timar' \\ Deiana Roman ${ }^{3}$ \\ Bogdan Timar ${ }^{2}$}

'Second Department of Internal Medicine, "Victor Babes" University of Medicine and Pharmacy, Timisoara, Romania; ${ }^{2}$ Department of Functional Sciences, "Victor Babes" University of Medicine and Pharmacy, Timisoara, Romania; ${ }^{3}$ Municipal Clinical Emergency Hospital, Timisoara, Romania
Correspondence: Mirela Frandes Department of Functional Sciences, "Victor Babes" University of Medicine and Pharmacy, 2 Eftimie Murgu, Timisoara 30004I, Romania

Tel +4073 III 7020

Fax +40256462856

Email mirela.frandes@umft.ro
Background: Dementia and cognitive dysfunction have many causes. There is strong evidence that diabetes mellitus (DM) increases the risk of cognitive impairment and dementia. Optimal glycemic control, identification of diabetic risk factors, and prophylactic approach are essential in the prevention of cognitive complications.

Aims: The main purpose of this study was to establish the cognitive impairment in DM patients, cared for in the Diabetes Center from Timisoara. Also, we investigated the prevalence of dementia in our group as well as the risk factors involved in the progression of mild cognitive impairment (MCI) to dementia.

Patients and methods: We considered a sample of 207 type 2 DM (T2DM) patients, aged between 33 and 81 years, mean $57.49( \pm 11.37)$ years. We established the diagnosis of dementia based on the Mini-Mental State Examination (MMSE) test, as well as on the psychological testing, psychiatric and neurological investigations, and imaging tests (computerized tomography and MRI).

Results: A percentage of $42.03 \%$ of patients presented MCI, mean age 63 (57.00-71.00) years, being older than patients without MCI, mean age 52.00 (45.00-61.00) years, $P<0.001$. We observed that diabetes duration was a significant risk factor for developing dementia. Also, patients with MCI presented higher values of body fat than patients without MCI. Moreover, we found that glucose levels, low-density lipoprotein cholesterol levels, the presence of stroke events, and the presence of cardiovascular disease were significant risk factors for MCI conversion to dementia. Conclusion: Patients with T2DM at early to severe stages of MCI are more likely to develop dementia and should be regularly evaluated for their cognitive status. Regular administrations of the MMSE test can be done to detect early stages of MCI development. Also, to reduce the progression of cognitive impairment to dementia, it is worthwhile to give greater importance to glycemic control and overall DM management.

Keywords: diabetes mellitus, metabolic syndrome, insulin resistance, cognitive impairment, dementia

\section{Introduction}

Diabetes mellitus (DM) is a worldwide major pathological condition due to its high prevalence, its negative impact on both lifespan and quality of life, as well as the extremely high costs it requires. An impressive dynamic growth in its prevalence on a global level is estimated in next years. If in 1980, there were around 108 million patients with DM, their number has increased to 422 million in 2016. ${ }^{1}$ The global prevalence of DM has almost doubled from 1980, a growth that can be explained by the increase of the DM risk factors' prevalence, such as overweight and obesity. A substantial 
increase of DM prevalence can be observed in poorly developed countries as well as in developing countries.,3

The various pathogenetic mechanisms involved in the appearance and progression of DM chronic complications are, in fact, the consequence of a single process, induced by hyperglycemia: anion superoxide overproduction $(\mathrm{O} 2)^{4,5}$ Intracellular hyperglycemia activates all major pathways of chronic complications: polyol pathway, increase in hexosamine activity, protein kinase $\mathrm{C}$ activation, advanced glycosylation end products, oxidative stress, and apoptosis, inflammation, expression and action of hormones, growth factors, cytokines and inflammatory mediators. ${ }^{6,7}$ The impact of DM and DM complications on the central nervous system have been less clear. ${ }^{8}$ Generally, DM has been associated to cognitive decline, especially in elderly people, apparition of cognitive impairment, and an increased risk of developing dementia, which includes both Alzheimer's disease (AD) and vascular dementia. ${ }^{9}$

Dementia is a devastating disease that most frequently appears at elderly age. However, several pathological conditions may favor or increase the apparition of cognitive decline at any age; around 4.6 million of new cases are estimated to occur worldwide every year, and the number of people affected by cognitive decline has been predicted to double every 20 years, 42 million by 2020 and 81 million by $2040 .^{10}$ DM and associated comorbidities increase the risk of cognitive impairment development as well as the risk of progression from such an impairment to dementia. Therefore, the prediction of cognitive decline development is an extremely important clinical factor.

Numerous DM-related mechanisms may accelerate the decline of cognitive functions as well as the progression to more advanced conditions, such as dementia. These mechanisms include hyperinsulinemia, insulin-resistant syndrome, disturbances of insulin homeostasis in the brain, generation of advanced products of glycosylation and interplay with the insulin-degrading enzyme, which is involved in both insulin and amyloid proteolysis. ${ }^{11}$ A cognitive decline induced by DM may also be influenced by generating both vascular disorders and impairment of the astrocytic trafficking and disruption of the neurovascular coupling. ${ }^{12}$ However, considering the expected increase in the global prevalence of DM at all ages, the risk of developing cognitive impairment as well as the progression to dementia may also increase. ${ }^{13-16}$

Dementia in type 2 DM (T2DM) may be associated with reduced physical and mental functions, chronic complications, and ultimately increased mortality. Nevertheless, apparition of cognitive impairment as well as its development to dementia may also be influenced by poor glycemic control, lipid disorder, generating decreased adherence to diet and hypoglycemic medication and higher health costs. Thus, early identification strategies of mild cognitive impairment (MCI) progression are of high clinical importance.

The primary purpose of this study was to establish the cognitive impairment in DM patients, cared for in the Center for Diabetes Treatment from Timisoara. At the same time, we determined the association between patients' medical characteristics and factors influencing the conversion to dementia in subjects with MCI over a follow-up period of 3 years in a prospective community-based study. Also, we investigated the prevalence of dementia in our group, and we compared the main risk factors involved in patients with dementia and those without dementia.

\section{Methods}

\section{Study design and patients}

In this study, we enrolled 207 patients diagnosed with T2DM attending scheduled visits in the Center for Diabetes Treatment of the "Pius Brinzeu" Emergency Hospital Timisoara, Romania. The protocol of our study was approved by the "Pius Brinzeu" Emergency Hospital Timisoara Ethics Committee. Also, the study was conducted according to the declaration of Helsinki, and all the patients included in the study were provided written informed consent for the acquisition, analysis, and publishing of the anonymized data collected during their hospital admission.

The exclusion criteria included the inability to provide informed consent, failure to provide accurate anamnestic medical data, the presence of major cardiovascular events (according to Hicks 2014 criteria), and absence of acute vascular events.

The enrolled patients were aged between 33 and 81 years, mean $57.49( \pm 11.37)$ years. Half of the patients were males $53.1 \%$, mean age 58.24 ( \pm 11.64$), 95 \%$ CI $(56.03,60.45)$, while $46.9 \%$ were females, mean age $56.67( \pm 10.94), 95 \%$ CI $(54.41,58.92)$. The flowchart of the study is presented in Figure 1.

\section{Clinical, anthropometric, and laboratory data}

The lipid profile, the presence of microvascular complications, and the presence of other comorbidities, such as chronic kidney disease, cardiovascular disease, were assessed for all the included patients.

Data regarding diabetes history were collected from the patients' medical records. We assessed the weight status 


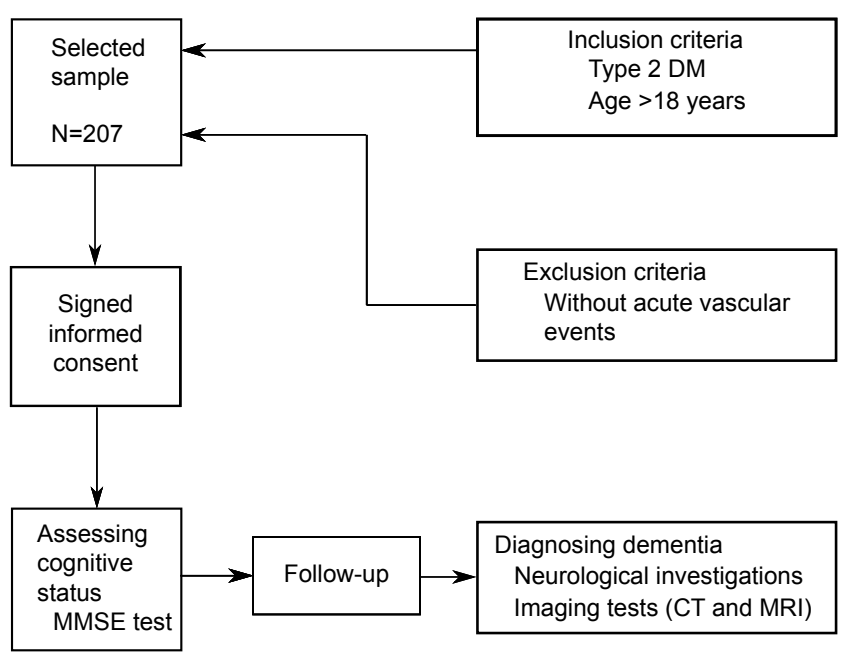

Figure I Study flow diagram.

Abbreviations: CT, computerized tomography; DM, diabetes mellitus; MMSE, Mini-Mental State Examination.

by measuring the body mass index (BMI), as the weight (expressed in kilograms) divided by the square of the height (expressed in meters). We considered the presence of hypertension when systolic blood pressure was $>140 \mathrm{mmHg}$ and/or diastolic blood pressure was higher $90 \mathrm{mmHg}$ or any value in patients under antihypertensive treatment. We considered dyslipidemia (disordered lipids in the blood) when total cholesterol was $>200 \mathrm{mg} / \mathrm{dL}$ and/or high-density lipoprotein cholesterol (HDLc) was $<45 \mathrm{mg} / \mathrm{dL}$ and/or low-density lipoprotein cholesterol (LDLc) was greater than $100 \mathrm{mg} / \mathrm{dL}$ and/or triglycerides were greater than $150 \mathrm{mg} / \mathrm{dL}$.

The HbA1c level was measured using an National Glycohemoglobin Standardization Program-standardized and Diabetes Control and Complications Trial-compliant immune-turbidimetric assay (Roche), including an intermeasurement coefficient of variation of $1.64 \%$, following the manufacturer's specifications. Triglyceride, HDLc, and total cholesterol levels were measured using an autoanalyzer (Cobas-Mira Roche) with enzymatic techniques. LDLc was calculated according to Friedewald formula when triglycerides levels were $<400 \mathrm{mg} / \mathrm{dL}$. The presence of chronic kidney disease was diagnosed following the Kidney Disease: Improving Global Outcomes 2012 guidelines. The diagnosis of retinopathy was established by performing a funduscopic examination by an ophthalmologist, specialist in diabetic eye complications.

\section{Cognitive impairment assessment}

Mini-Mental State Examination (MMSE) was used to assess the level of cognitive impairment. MMSE is one of the most widely employed screening tests for cognition impairment in the world. ${ }^{17}$
The standard MMSE form was administered to each patient. The scale of MMSE ranges from 0 to 30 points, where lower scores indicate poor cognitive performance, and higher scores indicate better cognitive performance. The MMSE form includes 19 questions conceived to evaluate the patient's mental status, being divided into the following 5 categories: 10 questions about orientation (year, season, date, day, month, state, city, close street, floor, and location), 2 items regarding memory (repeat the words car, window and vase, and after delayed recall), 1 item about calculation, 5 language items, and 1 constructional item (copy overlapping pentagons).

We used the Romanian version of standard MMSE test, ${ }^{18}$ considering the following cutoff scores for the classification of cognitive impairment: MMSE scores $\geq 27$ revealed a normal cognition, scores between 21 and 26 indicated MCI, scores between 11 and 20 reported moderate cognitive impairment, and scores lower than 10 were classified as severe cognitive impairment.

\section{Dementia assessment}

At follow-up, using psychiatric and neurological investigations, as well as imaging tests (computerized tomography and MRI), we have established the diagnosis of dementia. We considered the presence of specific signs, such as AD: medial temporal lobe atrophy and parietal atrophy; frontotemporal lobar degeneration: (asymmetric) frontal lobe atrophy and atrophy of the temporal pole; and vascular dementia: global atrophy, diffuse white matter lesions, as a positive diagnosis of dementia.

\section{Statistical analysis}

Collected data were presented as mean $( \pm \mathrm{SD})$ for continuous variables with a Gaussian distribution, median (IQR) for continuous variables without Gaussian distribution, or absolute frequency (percentage) for nominal variables. The normality of continuous variable distributions was tested using the Kolmogorov-Smirnov's test, while the equality of variances was tested using Levene's test.

The significance of the difference between groups was assessed by using the Student's $t$-test (means, Gaussian populations), Mann-Whitney $U$ test (medians, non-Gaussian populations), and Pearson's chi-squared or Fisher's exact test (proportions). The influence of one or more confounding factors in dichotomous outcomes was evaluated by deriving univariate and multivariate logistic regression models, while the goodness of fit was calculated using Nagelkerke's $R^{2}$ method. The linearity of the continuous variables with respect 
to the logit of the dependent variable was assessed by applying the Box-Tidwell procedure.

Data analysis was performed by using the SPSS v. 17 software (SPSS Inc., Chicago, IL, USA) and the R software packages (v.3.3) for statistical computing. We considered a $P$-value $<0.05$ as the threshold for statistical significance and a confidence level of 0.95 for estimating intervals.

\section{Results}

The baseline patients' characteristics are presented in Table 1.

We observed that $42.03 \%$ of patients presented MCI, mean age 63 (57.00-71.00) years, being older than patients without MCI, mean age 52.00 (45.00-61.00) years, $P<0.001$.

Table I Baseline characteristics of the patients

\begin{tabular}{|c|c|}
\hline Number of patients & 207 \\
\hline Age (years) ${ }^{\mathrm{a}}$ & $58.00(47.00-65.50)$ \\
\hline Gender (male) ${ }^{b}$ & $110(53.10 \%)$ \\
\hline Diabetes duration (years) ${ }^{\mathrm{a}}$ & $10.00(8.00-13.00)$ \\
\hline BMI $\left(\mathrm{kg} / \mathrm{m}^{2}\right)^{\mathrm{c}}$ & $28.00( \pm 5.175)$ \\
\hline $\mathrm{AC}(\mathrm{cm})^{\mathrm{c}}$ & $99.98( \pm 19.15)$ \\
\hline Smoking ${ }^{\mathrm{b}}$ & 64 (30.90\%) \\
\hline Alcoholb $^{b}$ & $44(21.30 \%)$ \\
\hline Fasting glycemia $(\mathrm{mg} / \mathrm{dL})^{\mathrm{a}}$ & $129.00(95.00-176.00)$ \\
\hline Postprandial glycemia $(\mathrm{mg} / \mathrm{dL})^{\mathrm{a}}$ & $164.50(110.00-216.00)$ \\
\hline $\mathrm{HbAlc}(\%)^{\mathrm{a}}$ & $8.20(7.00-9.40)$ \\
\hline Triglycerides $(\mathrm{mg} / \mathrm{dL})^{\mathrm{a}}$ & $132.00(97.00-195.50)$ \\
\hline Total cholesterol $(\mathrm{mg} / \mathrm{dL})^{\mathrm{a}}$ & $182.00(153.00-215.00)$ \\
\hline HDLc $(m g / d L)^{a}$ & $43.00(36.00-52.50)$ \\
\hline LDLc (mg/dL) $)^{\mathrm{a}}$ & $104.60(77.30-135.00)$ \\
\hline Non-HDLc (mg/dL) ${ }^{a}$ & $136.00(107.00-170.00)$ \\
\hline \multicolumn{2}{|l|}{$\mathrm{HBP}$} \\
\hline Normal & $56(27.10 \%)$ \\
\hline Prehypertension & $7(3.4 \%)$ \\
\hline Hypertension stage I & $66(31.9 \%)$ \\
\hline Hypertension stage II & $78(37.7 \%)$ \\
\hline Diabetic polyneuropathy ${ }^{\mathrm{b}}$ & $105(50.7 \%)$ \\
\hline \multicolumn{2}{|l|}{ Diabetic arteriopathy ${ }^{\mathrm{b}}$} \\
\hline Intermittent claudication & $6(2.9 \%)$ \\
\hline Rest pain & $15(7.2 \%)$ \\
\hline Ischemic ulcers or gangrene & $6(2.9 \%)$ \\
\hline \multicolumn{2}{|l|}{ Diabetic retinopathy ${ }^{b}$} \\
\hline Background retinopathy & $9(4.3 \%)$ \\
\hline Preproliferative retinopathy & $25(12.1 \%)$ \\
\hline Proliferative retinopathy & $5(2.4 \%)$ \\
\hline Gall bladder $^{\mathrm{b}}$ & $4 \mid(19.8 \%)$ \\
\hline Pancreatitis $^{\mathrm{b}}$ & $8(3.9 \%)$ \\
\hline Cardiovascular disease $^{\mathrm{b}}$ & $86(41.5 \%)$ \\
\hline Chronic kidney disease $^{b}$ & $67(32.4 \%)$ \\
\hline Stroke ${ }^{\mathrm{b}}$ & $34(16.4 \%)$ \\
\hline
\end{tabular}

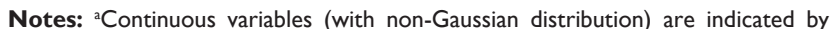
their median (IQR). ' $\mathrm{C}$ Categorical variables are presented by absolute frequency (percentage) in the sample. 'Continuous variables (with Gaussian distribution) are indicated by their mean (SD).

Abbreviations: AC, abdominal circumference; BMI, body mass index; DM, diabetes mellitus; HBP, high blood pressure; HDLc, high-density lipoprotein cholesterol; LDLc, low-density lipoprotein cholesterol.
In addition, we observed that patients with $\mathrm{MCI}$ presented higher values of BMI and abdominal circumference than patients without MCI, $29.00(27.00-32.40)$ vs 27.80 $(25.00-31.00) \mathrm{kg} / \mathrm{m}^{2}(P=0.030)$ and $105.00(92.00-115.00)$ vs $96.50(81.00-108.00) \mathrm{cm}(P=0.002)$, respectively (Table 2).

A higher percentage of patients with MCI presented hypertension when compared with those without MCI, $86.21 \%$ vs $63.33 \%(P<0.001)$. At the same time, a higher percentage of patients with MCI presented cardiovascular disease (coronary ischemic disease) when compared with those without MCI, $64.37 \%$ vs $25 \%(P<0.001)$. Also, a higher percentage of patients with MCI presented stroke events when compared with those without MCI, $25.28 \%$ vs $10 \%(P=0.003)$ (Table 2).

The presence of dementia was associated with higher age (72 vs 60 years; $P<0.001)$ and higher LDLc levels $(115.20$ vs $101.70 \mathrm{mg} / \mathrm{dL} ; P=0.045)$. At the same time, the presence of dementia was associated with a higher prevalence of cardiovascular disease $(100 \%$ vs $51.56 \% ; P<0.001)$ and stroke events $(47.83 \%$ vs $17.19 \% ; P=0.004)$. The detailed comparison between DM patients without MCI vs DM patients with MCI can be found in Table 3 .

\section{Univariate logistic regressions}

In the univariate logistic regression models, the effect of lipid parameters on the presence of dementia was analyzed by considering the average values of cholesterol (total cholesterol: non-HDLc, LDLc, and HDLc) as well as the average values of triglycerides. Additionally, we assessed the impact of glycemic parameters over the presence of dementia by considering the average values of both fasting glycemia and postprandial glycemia as well as HbA1c values.

We observed that age was not a risk factor for developing dementia (OR=0.969, 95\% CI: 0.963-0.976, $P<0.001$ ). However, we observed that diabetes duration was a significant risk factor (OR=1.421, 95\% CI: 1.261-1.601, $P<0.001$ ). Additionally, we observed that HDLc was a significant protective factor against the risk of MCI conversion to dementia (OR=0.984; 95\% CI: 0.939-0.992; $P<0.001)$. On the contrary, increased LDLc values were a significant risk factor for MCI conversion to dementia (OR=1.009; $95 \%$ CI: $1.001-1.017 ; P=0.027)$. Moreover, DM-related complications, such as polyneuropathy and retinopathy, were not significant risk factors for MCI conversion to dementia $(\mathrm{OR}=1.590 ; 95 \% \mathrm{CI}: 0.656-3.855 ; P=0.305$ and $\mathrm{OR}=0.083$; 95\% CI: $0.028-0.271 ; P=0.004$, respectively). Even more, we observed that the presence of cardiovascular disease also was 
Table 2 Comparison between groups: DM patients without $\mathrm{MCl}$ vs DM patients with $\mathrm{MCl}$

\begin{tabular}{|c|c|c|c|}
\hline & Without $\mathrm{MCl}$ & With $\mathrm{MCl}$ & $P$-value \\
\hline Number of patients & 120 & 87 & Not applicable \\
\hline Age (years) ${ }^{\mathrm{a}}$ & $52.00(45.00-61.00)$ & $63.00(57.00-71.00)$ & $<0.001$ \\
\hline Gender (male) ${ }^{b}$ & 55 (45.83\%) & $55(63.22 \%)$ & 0.013 \\
\hline Diabetes duration (years) ${ }^{\mathrm{a}}$ & $9.00(7.00-11.00)$ & $11.00(10.00-18.00)$ & $<0.001$ \\
\hline BMI $\left(\mathrm{kg} / \mathrm{m}^{2}\right)^{\mathrm{a}}$ & $28.00(25.00-32.00)$ & $29.00(27.00-32.00)$ & 0.168 \\
\hline $\mathrm{AC}(\mathrm{cm})^{\mathrm{a}}$ & $97.00(81.00-109.00)$ & $90.00(92.00-115.00)$ & 0.019 \\
\hline Smoking & $46(38.33 \%)$ & $18(20.68 \%)$ & 0.007 \\
\hline Alcoholb $^{b}$ & $23(19.17 \%)$ & $21(24.14 \%)$ & 0.388 \\
\hline Fasting glycemia $(\mathrm{mg} / \mathrm{dL})^{\mathrm{a}}$ & $124.00(95.00-171.00)$ & | 35.00 (93.00-177.00) & 0.774 \\
\hline Postprandial glycemia $(\mathrm{mg} / \mathrm{dL})^{\mathrm{a}}$ & $150.00(110.00-210.00)$ & $180.00(110.00-234.00)$ & $0.08 \mathrm{I}$ \\
\hline $\mathrm{HbAlc}(\%)^{\mathrm{a}}$ & $8.10(7.00-9.30)$ & $8.30(7.00-9.60)$ & 0.506 \\
\hline Triglycerides $(\mathrm{mg} / \mathrm{dL})^{\mathrm{a}}$ & $126.00(95.00-172.00)$ & 140.00 (109.00-242.00) & 0.070 \\
\hline Total cholesterol $(\mathrm{mg} / \mathrm{dL})^{\mathrm{a}}$ & $181.00(152.00-218.00)$ & $182.00(153.00-215.00)$ & 0.986 \\
\hline HDLc (mg/dL) ${ }^{a}$ & $44.00(37.00-56.00)$ & $41.00(34.00-50.00)$ & 0.177 \\
\hline LDLc $(\mathrm{mg} / \mathrm{dL})^{\mathrm{a}}$ & $104.20(74.60-135.00)$ & $106.20(80.20-135.60)$ & 0.895 \\
\hline Non-HDLc (mg/dL) ${ }^{a}$ & $135.00(102.00-172.00)$ & 140.00 (I I $2.00-170.00)$ & 0.536 \\
\hline Hypertension ${ }^{\mathrm{b}}$ & 76 (63.33\%) & 75 (86.2 I\%) & $<0.001$ \\
\hline Diabetic polyneuropathy ${ }^{b}$ & $57(47.50 \%)$ & $48(55.17 \%)$ & 0.276 \\
\hline Diabetic arteriopathy ${ }^{\mathrm{b}}$ & $12(10 \%)$ & $15(17.24 \%)$ & 0.127 \\
\hline Diabetic retinopathy ${ }^{\mathrm{b}}$ & $18(15 \%)$ & $21(24.14 \%)$ & 0.097 \\
\hline Gall bladder ${ }^{b}$ & $15(12.5 \%)$ & $26(29.88 \%)$ & 0.002 \\
\hline Pancreatitis $^{b}$ & $4(3.33 \%)$ & $4(4.60 \%)$ & $0.64 I$ \\
\hline Cardiovascular disease ${ }^{\mathrm{b}}$ & $30(25 \%)$ & $56(64.37 \%)$ & $<0.001$ \\
\hline Chronic kidney disease $^{b}$ & $33(27.5 \%)$ & $34(39.08 \%)$ & 0.079 \\
\hline Stroke ${ }^{b}$ & $12(10 \%)$ & $22(25.28 \%)$ & 0.003 \\
\hline
\end{tabular}

Notes: ${ }^{\mathrm{a} C o n t i n u o u s ~ v a r i a b l e s ~(w i t h ~ n o n-G a u s s i a n ~ d i s t r i b u t i o n) ~ a r e ~ i n d i c a t e d ~ b y ~ t h e i r ~ m e d i a n ~(I Q R) . ~}{ }^{\mathrm{b} C a t e g o r i c a l}$ variables are presented by percentage (absolute frequency) in the sample.

Abbreviations: AC, abdominal circumference; BMI, body mass index; DM, diabetes mellitus; HDLc, high-density lipoprotein cholesterol; LDLc, low-density lipoprotein cholesterol; $\mathrm{MCl}$, mild cognitive impairment.

not risk factor for developing dementia (OR $=0.365,95 \% \mathrm{CI}$ : $0.226-0.589, P=0.001)$. On the contrary, stroke events were a significant risk factor for dementia $(\mathrm{OR}=6.417,95 \% \mathrm{CI}$ : $2.538-16.222, P<0.001)$.

\section{Multivariate logistic regression}

Binomial logistic regression models were derived in order to assess the effects of the considered parameters on the likelihood that DM patients with MCI have developed dementia. We applied a Bonferroni correction resulting in statistical significance being accepted when $P<0.005$. Including this assessment, all continuous independent variables were linearly related to the logit of the dependent variable.

The resulted regression model was statistically significant, $\chi^{2}(2)=14.072, P<0.001$, explaining 68.9\% (Nagelkerke $R^{2}$ ) of the variance in the presence of dementia among T2DM with MCI. We found that six predictor variables were statistically significant: diabetes duration, average fasting glycemia, average $\mathrm{HbAlc}$, average HDLc, average LDLc, and the presence of stroke events (Table 4). Patients with longer diabetes duration had 1.542 times higher odds of developing dementia than patients with $\mathrm{MCI}$ and shorter diabetes duration $(\mathrm{OR}=1.542,95 \% \mathrm{CI}$ : $1.268-1.875)$. Higher HDLc values were associated with a decreased likelihood of developing dementia $(\mathrm{OR}=0.922$, 95\% CI: 0.854-0.997), but rising LDLc was associated with an increased likelihood of developing dementia (OR=1.018, 95\% CI: 1.004-1.039). Also, we noted that patients with at least one stroke event had 1.523 times higher odds to develop dementia than patients without stroke events (OR=1.523, 95\% CI: 1.401-1.704). Moreover, the presence of cardiovascular disease resulted in 1.258 times higher odds to develop dementia than patients without cardiovascular disease (Figure 2). 
Table 3 Comparison between groups: DM patients with $\mathrm{MCl}$ who did not develop dementia vs DM patients with $\mathrm{MCl}$ who developed dementia

\begin{tabular}{|c|c|c|c|}
\hline & Without dementia & With dementia & $P$-value ${ }^{c}$ \\
\hline Number of patients & 64 & 23 & Not applicable \\
\hline Age (years) ${ }^{\mathrm{a}}$ & $60.00(54.00-65.00)$ & $72.00(69.00-76.00)$ & $<0.001$ \\
\hline Gender (male) $^{\mathrm{b}}$ & 40 (62.50\%) & $15(65.21 \%)$ & 0.817 \\
\hline Diabetes duration (years) $^{a}$ & II.00 (9.00-II.50) & $29.00(22.00-31.00)$ & $<0.001$ \\
\hline BMI $\left(\mathrm{kg} / \mathrm{m}^{2}\right)^{\mathrm{a}}$ & $30.50(27.00-33.00)$ & $27.50(25.50-29.00)$ & 0.009 \\
\hline $\mathrm{AC}(\mathrm{cm})^{\mathrm{a}}$ & $108.00(97.00-117.00)$ & $94.00(85.00-107.50)$ & 0.011 \\
\hline Smoking ${ }^{\mathrm{b}}$ & $16(25 \%)$ & $2(8.7 \%)$ & 0.098 \\
\hline Alcohol $^{b}$ & $18(28.12 \%)$ & $3(13.04 \%)$ & 0.147 \\
\hline Fasting glycemia $(\mathrm{mg} / \mathrm{dL})^{\mathrm{a}}$ & $129.00(87.00-175.00)$ & $166.00(\mid 22.00-186.50)$ & 0.041 \\
\hline Postprandial glycemia $(\mathrm{mg} / \mathrm{dL})^{\mathrm{a}}$ & $173.00(87.00-175.00)$ & $189.00(153.00-249.00)$ & 0.182 \\
\hline $\mathrm{HbAlc}(\%)^{\mathrm{a}}$ & $8.45(7.00-9.80)$ & $7.90(7.10-9.10)$ & 0.400 \\
\hline Triglycerides $(\mathrm{mg} / \mathrm{dL})^{\mathrm{a}}$ & I40.00 (I I I0.00-242.00) & I37.00 (94.50-244.00) & 0.593 \\
\hline Total cholesterol $(\mathrm{mg} / \mathrm{dL})^{\mathrm{a}}$ & $182.00(|53.00-2| 1.00)$ & $194.00(164.00-241.50)$ & 0.240 \\
\hline HDLc (mg/dL) ${ }^{a}$ & $40.00(34.00-52.00)$ & $43.00(35.00-49.00)$ & 0.950 \\
\hline LDLc $(\mathrm{mg} / \mathrm{dL})^{\mathrm{a}}$ & $101.70(79.40-125.00)$ & II $15.20(94.60-149.50)$ & 0.045 \\
\hline Non-HDLc (mg/dL) ${ }^{\mathrm{a}}$ & I37.00 (I08.00-I66.00) & $140.00(124.50-193.00)$ & 0.147 \\
\hline Hypertension $^{\mathrm{b}}$ & $54(84.3 \%)$ & 21 (9l.3\%) & 0.408 \\
\hline Diabetic polyneuropathy ${ }^{\mathrm{b}}$ & $34(53.12 \%)$ & $14(60.86 \%)$ & 0.522 \\
\hline Diabetic arteriopathy ${ }^{\mathrm{b}}$ & $9(14.06 \%)$ & $6(26.08 \%)$ & 0.190 \\
\hline Diabetic retinopathy ${ }^{\mathrm{b}}$ & $18(28.12 \%)$ & $3(13.04 \%)$ & 0.147 \\
\hline Gall bladder $^{\mathrm{b}}$ & $19(29.68 \%)$ & $7(30.43 \%)$ & 0.946 \\
\hline Pancreatitis $^{\mathrm{b}}$ & $4(6.25 \%)$ & $0(0 \%)$ & 0.220 \\
\hline Cardiovascular disease $^{b}$ & $33(51.56 \%)$ & $23(100 \%)$ & $<0.001$ \\
\hline Chronic kidney disease $^{\mathrm{b}}$ & $24(37.5 \%)$ & $10(43.47 \%)$ & 0.614 \\
\hline Stroke $^{b}$ & II (I7.19\%) & II (47.83\%) & 0.004 \\
\hline
\end{tabular}

Notes: ${ }^{\mathrm{a} C o n t i n u o u s ~ v a r i a b l e s ~(w i t h ~ n o n-G a u s s i a n ~ d i s t r i b u t i o n) ~ a r e ~ i n d i c a t e d ~ b y ~ t h e i r ~ m e d i a n ~(I Q R) . ~}{ }^{\mathrm{b} C}$ ategorical variables are presented by absolute frequency (percentage) in the sample. ${ }^{C P}$-value was computed using Mann-Whitney $U$ test for continuous variables (with non-Gaussian distribution) and Pearson's chi-squared test for nominal variables.

Abbreviations: AC, abdominal circumference; BMI, body mass index; DM, diabetes mellitus; HDLc, high-density lipoprotein cholesterol; LDLc, low-density lipoprotein cholesterol; $\mathrm{MCl}$, mild cognitive impairment.

\section{Discussion}

Our study showed that conversion to dementia in T2DM patients with MCI is significantly influenced by the association of diabetes duration, glycemic control (fasting glycemia,

Table 4 Predictors of developing dementia in patients with DM and cognitive impairment (multivariate logistic regression model; Nagelkerke $R^{2}=0.689$ )

\begin{tabular}{|c|c|c|c|}
\hline \multirow[t]{2}{*}{ Predictor variable } & \multirow[t]{2}{*}{ OR } & \multicolumn{2}{|l|}{$95 \% \mathrm{Cl}$} \\
\hline & & Lower & Upper \\
\hline Age (years) & 0.956 & 0.885 & 1.033 \\
\hline Diabetes duration (years)* & 1.542 & 1.268 & 1.875 \\
\hline Fasting glycemia $(\mathrm{mg} / \mathrm{dL})^{*}$ & 1.019 & 1.002 & 1.035 \\
\hline Postprandial glycemia (mg/dL) & 1.008 & 0.990 & 1.026 \\
\hline $\mathrm{HbAlc} \mathrm{( \% )*}$ & 0.387 & 0.257 & 0.450 \\
\hline Triglycerides (mg/dL) & 0.984 & 0.966 & 1.002 \\
\hline HDLc (mg/dL)* & 0.922 & 0.854 & 0.997 \\
\hline LDLc (mg/dL)* & 1.018 & 1.004 & 1.039 \\
\hline Stroke ${ }^{\mathrm{a}, *}$ & 1.523 & $\mathrm{I} .40 \mathrm{I}$ & $\mathrm{I} .704$ \\
\hline Cardiovascular disease & 1.258 & 1.103 & $\mathrm{I} .347$ \\
\hline
\end{tabular}

Notes: aPresence of at least one stroke event. *Predictor variable is significant both independently and as a cofactor.

Abbreviations: DM, diabetes mellitus; HDLc, high-density lipoprotein cholesterol; LDLc, low-density lipoprotein cholesterol.
HbA1c), cholesterol level, the apparition of stroke events, and cardiovascular disease. Thus, besides the presence of T2DM per se, other factors influence the decline to dementia over time. We observed that the rate of both hypo- and hyperglycemia events are higher in case of DM patients with dementia, $4.3 \%$ vs $1.1 \%$ and $65.2 \%$ vs $43.5 \%$, respectively. Similar studies also indicated that cognitive dysfunction with its wide range, from cognitive impairment to dementia, might be one of the complications of DM. Generally, both $\mathrm{DM}$ and cognitive dysfunction occur more commonly at an older age. However, T2DM might increase the risk of dementia at all ages in the form of multi-infarct dementia, $\mathrm{AD}$, or mixed type dementia. ${ }^{19}$ In addition, it was observed that $\mathrm{DM}$ is more closely correlated with vascular dementia $(100 \%-160 \%)$ when compared to $\mathrm{AD}(45 \%-90 \%){ }^{20}$

Our results suggested that the presence of DM might increase the long-term risk of dementia as well as the risk of cognitive impairment conversion to dementia. In addition, the risk of $\mathrm{AD}$ or dementia might also increase even in the prediabetic state without being directly related to the future development of DM. ${ }^{21}$ However, $\sim 80 \%$ of people with $\mathrm{AD}$ 

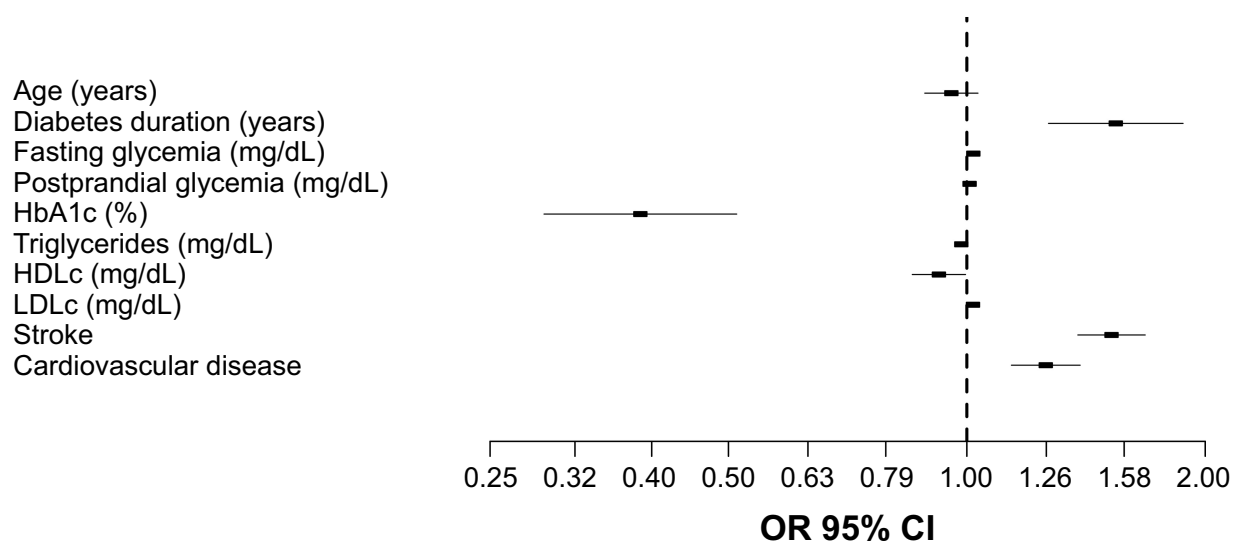

Figure 2 Multivariate risk analysis for developing dementia in T2DM patients with $\mathrm{MCl}$. The risk is expressed per I percentage point increase for $\mathrm{HbAlc}$, and I mg/dL point increase for fasting glycemia, postprandial glycemia, triglycerides, HDLc, and LDLc. For stroke events, and cardiovascular disease, the risk is expressed as dichotomous variables.

Abbreviations: HDLc, high-density lipoprotein cholesterol; LDLc, low-density lipoprotein cholesterol; MCI, mild cognitive impairment; T2DM, type 2 diabetes mellitus.

may also have impaired fasting glucose or DM.22 Also, DM accelerates the deterioration of cognition even faster than for non-DM elderly people. ${ }^{23}$ Even more, DM was associated with a risk of stroke increased by 1.15 times $(95 \% \mathrm{CI}$ : 1.08-1.23) for every $1 \%$ increase in $\mathrm{HbAl}^{24}$ and a risk of cerebrovascular accidents increased by $1.5-2$-fold..$^{25}$

In the recent years, the relation of DM to memory disorders has been investigated. It was found that T2DM was associated with decreases in processing speed, psychomotor speed, verbal memory, complex motor functioning, immediate recall, delayed recall, verbal fluency, visual retention, and attention. ${ }^{26-29}$ In addition, DM patients presented slower walking speed, lack of balance and increased falls, but it is not clear if the impact of DM on cerebral functions contributed to the apparition of these symptoms.

Similar to our findings, it was shown that cardiovascular disease increases the odds of developing dementia. ${ }^{30}$ In addition, stroke and subclinical cardiovascular and atherosclerosis markers are related to decline of cognitive functions in elderly patients with T2DM. ${ }^{31}$ In our group, MCI was diagnosed in $42.03 \%$ of patients. In a different study, the association between DM and cognitive impairment was evaluated, resulting that subjects with T2DM presented lower MMSE score than those without DM..$^{32}$ At the same time, increased odds of cognitive decline were related to the presence of DM when cognitive dysfunction was determined by MMSE score (OR=1.9; 95\% CI: 1.01-3.6). Other study investigated whether vascular and degenerative lesions in the brain mediate the association of DM with poor cognitive performance and to which extent. It was observed that DM patients' speed of processing and executive functions as well as memory function were significantly lower than in control group. ${ }^{33}$ Neuroimaging and neuropathological studies have also confirmed the role of DM in neurodegeneration. More exactly, T2DM was found to be strongly related to brain atrophy, ${ }^{34}$ which generally develops up to 3 times faster than in normal aging. ${ }^{35,36}$ Additionally, T2DM patients presented increased incidence of $\mathrm{AD},{ }^{37-39}$ and increased incidence of vascular dementia. Also, it was observed that $17.5 \%$ of elderly T2DM patients presented moderate to severe deficiency in daily activities, ${ }^{40,41} 11.3 \%$ presented impairment of cognitive functions, and $14.2 \%$ presented depression, ${ }^{42}$ which also negatively influence the cognitive functions and daily activities. ${ }^{43}$

We found that a heterogeneous disorder, such as T2DM, is accompanied by numerous comorbidities, like hypertension, stroke events, and dyslipidemia, where each has adverse effects on cognitive functions. Analyzing the presence of cardiovascular risk factors in patients with and without MCI, we found that hypertension was present in $86.21 \%$ of patients with MCI and $63.33 \%$ in those without $\mathrm{MCI}(P<0.001)$. The presence of dementia was associated with higher diabetes duration (29 vs 11 years; $P<0.001$ ), higher LDLc levels (115.20 vs $101.70 \mathrm{mg} / \mathrm{dL} ; P=0.045$ ).

All of the studies mentioned above come to support our findings and are in accord with them. Moreover, the strength of our study lies in the fact that it is the first of its kind conducted on the Romanian population, which has a particular socioeconomic climate that could also impact cognitive function. Also, due to the particularities of DM management in Romania, other studies and their findings might not necessarily reflect the situation encountered in this population.

Because the present study is a short-period prospective one, we must keep in mind its limitative character, rendered by the fact that past DM-related factors that might have influenced cognition cannot be quantified or assessed. Another 
limitation of this study is the fact that it is monocentric, so one could argue that beyond national characteristics we can find regional ones such as genetic, educational, traditional, culinary, and socioeconomical.

It becomes clear that patients with T2DM should regularly have their cognitive function evaluated. The early implementation of MMSE, which is a simple testing method, can lead to the detection of dementia in early stages. This examination might be an essential tool to access the ability of patients to understand their disease and treatment. Also, a prophylactic approach in conjunction with an optimal glycemic control and identification of diabetic risk factors are essential in the prevention of cognitive complications.

Although a lot of research has been conducted on the impact of DM on cognitive functions, many questions still remain. Regarding the Romanian population, a multi-centric study must be done to ensure a representative pool and act accordingly. Future studies will be essential to pinpoint risk factors for cognitive dysfunction and shed light on its ties to diabetes.

\section{Conclusion}

It is well known that in T2DM patients, we have a higher risk of strokes and cardiovascular events, influenced HbA1c and fasting glucose levels, dyslipidemia including higher LDLc levels, all of which are risk factors in developing cognitive impairment. All these risk factors are modifiable and can be kept under control by ensuring proper diabetes care by the health care specialist, by teaching patients how to manage their diabetes best and engage in self-care activities, as well as by performing regular cognitive function testing. We must also keep in mind that a deterioration in cognitive function will lead to poor self-care and compliance to diabetes treatment, decreasing the overall diabetes prognosis and increasing the chance of complications.

\section{Acknowledgment}

The authors gratefully acknowledge the professionals from the Center for Diabetes Treatment of the "Pius Brinzeu" Emergency Hospital Timisoara, Romania, for their support in data acquisition.

\section{Author contributions}

$\mathrm{OA}, \mathrm{MF}$, and BT conceived and designed the experiments and analyzed the data. OA, BT, and RT performed the experiments, OA, MF, BT, and DR contributed reagents/materials/ analysis tools. All authors contributed toward data analysis, drafting and critically revising the paper, gave final approval of the version to be published, and agreed to be accountable for all aspects of the work.

\section{Disclosure}

The authors report no conflicts of interest in this work.

\section{References}

1. Wild S, Roglic G, Green A, Sicree R, King H. Global prevalence of diabetes: estimates for the year 2000 and projections for 2030. Diabetes Care. 2004;27(5):1047-1053.

2. NCD Risk Factor Collaboration (NCD-RisC). Worldwide trends in diabetes since 1980: a pooled analysis of 751 population-based studies with 4.4 million participants. Lancet. 2016;387(10027): 1513-1530.

3. Global Burden of Metabolic Risk Factors for Chronic Diseases Collaboration. Cardiovascular disease, chronic kidney disease, and diabetes mortality burden of cardiometabolic risk factors from 1980 to 2010: a comparative risk assessment. Lancet Diabetes Endocrinol. 2014;2(8): 634-647.

4. Candido R, Forbes JM, Thomas MC, et al. A breaker of advanced glycation end products attenuates diabetes-induced myocardial structural changes. Circ Res. 2003;92(7):785-792.

5. Goldin A, Beckman JA, Schmidt AM, Creager MA. Advanced glycation end products: sparking the development of diabetic vascular injury. Circulation. 2006;114(6):597-605.

6. Yao D, Taguchi T, Matsumura T, et al. High glucose increases angiopoietin-2 transcription in microvascular endothelial cells through methylglyoxal modification of mSin3A. J Biol Chem. 2007; 282(42):31038-31045.

7. Liu H, Yu S, Xu W, Xu J. Enhancement of 26S proteasome functionality connects oxidative stress and vascular endothelial inflammatory response in diabetes mellitus. Arterioscler Thromb Vasc Biol. 2012;32(9): 2131-2140.

8. Arvanitakis Z, Wilson RS, Li Y, Aggarwal NT, Bennett DA. Diabetes and function in different cognitive systems in older individuals without dementia. Diabetes Care. 2006;29(3):560-565.

9. Biessels GJ, Staekenborg S, Brunner E, Brayne C, Scheltens P. Risk of dementia in diabetes mellitus: a systematic review. Lancet Neurol. 2006;5(1):64-74

10. Ferri CP, Prince M, Brayne C, et al. Global prevalence of dementia: a Delphi consensus study. Lancet. 2005;366(9503):2112-2117.

11. Shuba $\mathrm{N}$ and Karan. Assessment of the cognitive status in diabetes mellitus. J Clin Diagn Res. 2012;6(10):1658.

12. Zimmet P, Alberti KG, Shaw J. Global and societal implications of the diabetes epidemic. Nature. 2001;414(6865):782-787.

13. Mogi M, Horiuchi M. Neurovascular coupling in cognitive impairment associated with diabetes mellitus. Circ J. 2011;75(5):1042-1048.

14. Velayudhan L, Poppe M, Archer N, Proitsi P, Brown RG, Lovestone S. Risk of developing dementia in people with diabetes and mild cognitive impairment. Br J Psychiatry. 2010;196(1):36-40.

15. Ciudin A, Espinosa A, Simó-Servat O, et al. Type 2 diabetes is an independent risk factor for dementia conversion in patients with mild cognitive impairment. J Diabetes Complications. 2017;31(8):1272-1274.

16. Simó R, Ciudin A, Simó-Servat O, Hernández C. Cognitive impairment and dementia: a new emerging complication of type 2 diabetes - the diabetologist's perspective. Acta Diabetologica. 2017;54(5):417-424.

17. Zimmet $\mathrm{P}$, Alberti KG, Shaw J. Global and societal implications of the diabetes epidemic. Nature. 2001;414(6865):782-787.

18. Cullen B, Fahy S, Cunningham CJ, et al. Screening for dementia in an Irish community sample using MMSE: a comparison of norm-adjusted versus fixed cut-points. Int J Geriatr Psychiatry. 2005;20(4):371-376.

19. Folstein MF, Folstein SE, McHugh PR. "Mini-mental state". A practical method for grading the cognitive state of patients for the clinician. J Psychiatr Res. 1975;12(3):189-198. 
20. Kravitz E, Schmeidler J, Schnaider Beeri M. Type 2 diabetes and cognitive compromise. Endocrinol Metab Clin North Am. 2013;42(3): 489-501.

21. Biessels GJ, Strachan MW, Visseren FL, Kappelle LJ, Whitmer RA. Dementia and cognitive decline in type 2 diabetes and prediabetic stages: towards targeted interventions. Lancet Diabetes Endocrinol. 2014; 2(3):246-255.

22. Janson J, Laedtke T, Parisi JE, O'Brien P, Petersen RC, Butler PC. Increased risk of type 2 diabetes in Alzheimer disease. Diabetes. 2004; 53(2):474-481.

23. Ravona-Springer R, Moshier E, Schmeidler J, et al. Changes in glycemic control are associated with changes in cognition in non-diabetic elderly. J Alzheimers Dis. 2012;30(2):299-309.

24. Selvin E, Bolen S, Yeh H-C, et al. Cardiovascular outcomes in trials of oral diabetes medications. Arch Internal Med. 2008;168(19):2070.

25. Folsom AR, Rasmussen ML, Chambless LE, et al. Prospective associations of fasting insulin, body fat distribution, and diabetes with risk of ischemic stroke. The Atherosclerosis Risk in Communities (ARIC) Study Investigators. Diabetes Care. 1999;22(7):1077-1083.

26. Mccrimmon RJ, Ryan CM, Frier BM. Diabetes and cognitive dysfunction. Lancet. 2012;379(9833):2291-2299.

27. Munshi M, Grande L, Hayes M, et al. Cognitive dysfunction is associated with poor diabetes control in older adults. Diabetes Care. 2006; 29(8):1794-1799.

28. Sinclair AJ, Girling AJ, Bayer AJ. Cognitive dysfunction in older subjects with diabetes mellitus: impact on diabetes self-management and use of care services. All Wales Research into Elderly (AWARE) Study. Diabetes Res Clin Pract. 2000;50(3):203-212.

29. Alencar RC, Cobas RA, Gomes MB. Assessment of cognitive status in patients with type 2 diabetes through the mini-mental status examination: a cross-sectional study. Diabetol Metab Syndr. 2010;2(1):10.

30. Wessels AM, Lane KA, Gao S, Hall KS, Unverzagt FW, Hendrie HC. Diabetes and cognitive decline in elderly African Americans: a 15-year follow-up study. Alzheimers Demen. 2011;7(4):418-424.

31. Feinkohl I, Keller M, Robertson CM, et al. Clinical and subclinical macrovascular disease as predictors of cognitive decline in older patients with type 2 diabetes: the Edinburgh type 2 diabetes study. Diabetes Care. 2013;36(9):2779-2786.
32. Zhao Q, Roberts RO, Ding D, et al. Diabetes is associated with worse executive function in both Eastern and Western populations: Shanghai aging study and mayo clinic study of aging. J Alzheimers Dis. 2015;47(1): $167-176$.

33. Qiu C, Sigurdsson S, Zhang Q, et al. Diabetes, markers of brain pathology and cognitive function. Ann Neurol. 2014;75(1):138-146.

34. Moran C, Phan TG, Chen J, et al. Brain atrophy in type 2 diabetes: regional distribution and influence on cognition. Diabetes Care. 2013;36(12): 4036-4042.

35. Kooistra M, Geerlings MI, van der Graaf Y, et al. Vascular brain lesions, brain atrophy, and cognitive decline. The Second Manifestations of ARTerial disease-magnetic resonance (SMART-MR) study. Neurobiol Aging. 2014;35(1):35-41.

36. van Elderen SG, de Roos A, de Craen AJ, et al. Progression of brain atrophy and cognitive decline in diabetes mellitus: a 3-year follow-up. Neurology. 2010;75(11):997-1002.

37. Whitmer RA. Type 2 diabetes and risk of cognitive impairment and dementia. Curr Neurol Neurosci Rep. 2007;7(5):373-380.

38. Guariguata L, Whiting DR, Hambleton I, Beagley J, Linnenkamp U, Shaw JE. Global estimates of diabetes prevalence for 2013 and projections for 2035. Diabetes Res Clin Pract. 2014;103(2):137-149.

39. Albai O, Roman D, Frandes M. Hypertriglyceridemia, an important and independent risk factor for acute pancreatitis in patients with type 2 diabetes mellitus. Ther Clin Risk Manag. 2017;13:515-522.

40. Bruce DG, Casey GP, Grange V, et al. Cognitive impairment, physical disability and depressive symptoms in older diabetic patients: the Fremantle Cognition in Diabetes Study. Diabetes Res Clin Pract. 2003; 61(1):59-67.

41. Watson GS, Craft S. The role of insulin resistance in the pathogenesis of Alzheimer's disease: implications for treatment. CNS Drugs. 2003; 17(1):27-45.

42. Yaffe K, Blackwell T, Whitmer RA, Krueger K, Barrett Connor E. Glycosylated hemoglobin level and development of mild cognitive impairment or dementia in older women. J Nutr Health Aging. 2006;10(4): 293-295.

43. Kodl CT, Seaquist ER. Cognitive dysfunction and diabetes mellitus. Endocr Rev. 2008;29(4):494-511.
Neuropsychiatric Disease and Treatment

\section{Publish your work in this journal}

Neuropsychiatric Disease and Treatment is an international, peerreviewed journal of clinical therapeutics and pharmacology focusing on concise rapid reporting of clinical or pre-clinical studies on a range of neuropsychiatric and neurological disorders. This journal is indexed on PubMed Central, the 'PsycINFO' database and CAS,

\section{Dovepress}

and is the official journal of The International Neuropsychiatric Association (INA). The manuscript management system is completely online and includes a very quick and fair peer-review system, which is all easy to use. Visit http://www.dovepress.com/testimonials.php to read real quotes from published authors. 